\title{
Generalizations of the Weak Law of the Excluded Middle
}

\author{
Andrea Sorbi and Sebastiaan A. Terwijn
}

\begin{abstract}
We study a class of formulas generalizing the weak law of the excluded middle and provide a characterization of these formulas in terms of Kripke frames and Brouwer algebras. We use these formulas to separate logics corresponding to factors of the Medvedev lattice.
\end{abstract}

\section{The Weak Law of the Excluded Middle}

Let IPC denote the intuitionistic propositional calculus. The weak law of the excluded middle (w.l.e.m. for short) is the principle

$$
\neg p \vee \neg \neg p .
$$

We view this as an axiom schema, in which we can substitute any formula for the variable $p$. Consider the logic IPC $+\neg p \vee \neg \neg p$, that is, the closure under deductions and substitutions of IPC and the w.l.e.m. The logic IPC $+\neg p \vee \neg \neg p$ has been studied extensively and is known in the literature under various names. It has been called

- the logic of the weak law of the excluded middle by Jankov,

- Jankov logic by various Russian authors,

- De Morgan logic by various American authors,

- testability logic by some others, and

- KC by still many others.

The term principle of testability for $\neg p \vee \neg \neg p$ goes back to Brouwer himself. In [3, p. 80] he writes (our comments in brackets):

Another corollary of the simple principle of the excluded third [i.e., $\tau \vee \neg \tau$ ] is the simple principle of testability, saying that every assignment $\tau$ of a property to a mathematical entity can be tested, i.e. proved to be either non-contradictory $[\neg \neg \tau]$ or absurd $[\neg \tau]$. (p. 80)

Received November 28, 2011; accepted January 27, 2013

2010 Mathematics Subject Classification: Primary 03B55, 03G10; Secondary 03D30

Keywords: weak law of the excluded middle, Brouwer algebras, Medvedev degrees

(C) 2015 by University of Notre Dame 10.1215/00294527-2864325 
Apparently the name KC comes from Dummett and Lemmon [5], who used LC to denote the "linear calculus," and K alphabetically precedes L, hence the name KC. The name De Morgan logic derives from the fact that the principle $\neg p \vee \neg \neg p$ is equivalent (over IPC) to the validity of the familiar De Morgan laws, or more precisely, to the only intuitionistically problematic implication $\neg(p \wedge q) \rightarrow \neg p \vee \neg q$ of these laws.

In this paper we will study the following sequence $\left\{\varphi_{k}\right\}_{k \geq 1}$ of formulas generalizing the w.l.e.m.

Definition 1.1 Let $\varphi_{1}=\neg p \vee \neg \neg p$, and for every $k>1$ define

$$
\varphi_{k}=\bigvee_{i \neq j}\left(\neg p_{i} \rightarrow \neg p_{j}\right) \vee \neg\left(\neg p_{1} \wedge \cdots \wedge \neg p_{k}\right)
$$

(where $1 \leq i, j \leq k$ ).

Notice that the formula $\varphi_{1}$ can be seen as a special case of $\varphi_{k}$ : indeed, $\varphi_{k}$ is equivalent over IPC to

$$
\neg p_{1} \vee \cdots \vee \neg p_{k} \vee \bigvee_{i \neq j}\left(\neg p_{i} \rightarrow \neg p_{j}\right) \vee \neg\left(\neg p_{1} \wedge \cdots \wedge \neg p_{k}\right)
$$

because $\neg p_{i}$ implies $\neg p_{j} \rightarrow \neg p_{i}$ in IPC. Then $\varphi_{1}$ is the special case $k=1$.

Also note that IPC proves $\varphi_{k} \rightarrow \varphi_{k+1}$ for every $k \geq 1$. This follows for example from Theorem 2.4, or from Theorem 3.3 below.

Below, we will study the logics IPC $+\varphi_{k}$, which again is the deductive closure of IPC and the axiom schema $\varphi_{k}$. In particular, IPC $+\varphi_{k}$ proves any substitution instance of $\varphi_{k}$.

\section{Kripke Semantics}

In this section we characterize the formulas $\varphi_{k}$ in (1) in terms of Kripke frames, and relate them to a class of formulas introduced by Smorynski [12].

We briefly recall some elementary notions about Kripke semantics. For unexplained terminology about Kripke frames and models, we refer the reader to Chagrov and Zakharyaschev [4] or Gabbay [7, p. 67].

A Kripke frame $\langle K, R\rangle$ is a nonempty set $K$, partially ordered by an accessibility relation $R$. Throughout this paper, we will work with Kripke frames that have a root, that is, a least element with respect to $R$, though this is not standardly part of the definition. As usual, we distinguish between models and frames. A Kripke model $\langle K, R, V\rangle$ is a Kripke frame together with a valuation $V$, which associates with every variable $p$ a set $V(p) \subseteq K$, such that if $x \in V(p)$ and $x R y$, then $y \in V(p)$ for every $x$ and $y$. Now the forcing relation $x \Vdash \chi$, with $x \in K$ and $\varphi$ a formula, is defined by

- $x \Vdash p$ if $x \in V(p)$;

- $x \Vdash \varphi \wedge \psi$ if and only if $x \Vdash \varphi$ and $x \Vdash \psi$;

- $x \Vdash \varphi \vee \psi$ if and only if $x \Vdash \varphi$ or $x \Vdash \psi$;

- $x \Vdash \varphi \rightarrow \psi$ if and only if for every $y$ with $x R y$, if $y \Vdash \varphi$, then $y \Vdash \psi$;

- $x \Vdash \neg \varphi$ if and only if there is no $y$ with $x R y$ and $y \Vdash \varphi$.

A formula $\varphi$ holds in a frame $K$, denoted by $K \models \varphi$, if $K \Vdash \varphi$ (meaning that $x \Vdash \varphi$ for every $x \in K$ ), for every valuation $V$ on the frame. A logic $\mathrm{L}$ is complete with respect to, or characterizes, a class of frames $\mathcal{K}$ if a formula is derivable in $\mathrm{L}$ if and only if it holds on every frame in $\mathcal{K}$. 
Definition 2.1 A Kripke frame with accessibility relation $R$ has topwidth $k$ if it has $k$ maximal nodes $x_{1}, \ldots, x_{k}$ such that for every $y \in K$, there is an $i$ with $y R x_{i}$.

Following Jankov [9], Gabbay [7, p. 67] showed that the logic IPC $+\neg p \vee \neg \neg p$ is complete with respect to the class of Kripke frames of topwidth 1. Smorynski [12] introduced, for every $k \geq 1$, the formula

$$
\sigma_{k}=\bigwedge_{0 \leq i<j \leq k} \neg\left(\neg p_{i} \wedge \neg p_{j}\right) \rightarrow \bigvee_{0 \leq i \leq k}\left(\neg p_{i} \rightarrow \bigvee_{j \neq i} \neg p_{j}\right)
$$

and showed that the logic IPC $+\sigma_{k}$ characterizes the class of Kripke frames of topwidth at most $k$ (henceforth we refer to this result as Smorynski's completeness theorem [12, Theorem 1.III.16]). In particular, IPC proves the formula $\sigma_{k} \rightarrow \sigma_{k+1}$ and IPC $+\sigma_{1}$ coincides with the logic of the w.l.e.m. Note that $\varphi_{k}$ has $k$ variables and that $\sigma_{k}$ has $k+1$. The relation between these formulas is sorted out below.

We now turn to a characterization of the formulas $\varphi_{k}$ in (1) in terms of Kripke frames. We start with some preliminaries about canonical models. For more on canonical models we refer to [4]. The canonical model $K$ of a logic L containing IPC consists of tableaux, that is, pairs $(\Gamma, \Delta)$ of sets of formulas, with the following properties. (Gabbay [7] uses saturated sets of formulas to define the canonical model, which is similar but different.)

(i) $(\Gamma, \Delta)$ is consistent with $\mathrm{L}$, meaning that for no $\chi_{1}, \ldots, \chi_{n} \in \Delta$, $\Gamma$ proves $\chi_{1} \cdots \chi_{n}$ over $\mathrm{L}$

(ii) $(\Gamma, \Delta)$ is maximal in the sense that $\Gamma \cup \Delta$ is the set of all formulas.

The accessibility relation $R$ in the canonical model is defined by

$$
(\Gamma, \Delta) R\left(\Gamma^{\prime}, \Delta^{\prime}\right) \Longleftrightarrow \Gamma \subseteq \Gamma^{\prime} \Longleftrightarrow \Delta \supseteq \Delta^{\prime} .
$$

This defines the canonical frame, and to make it into a model it is defined that every atomic formula in $\Gamma$ is forced in the node $(\Gamma, \Delta)$. It is a basic property of $K$ that for every node $(\Gamma, \Delta)$ and every formula $\varphi$,

$$
(\Gamma, \Delta) \Vdash \varphi \Longleftrightarrow \varphi \in \Gamma .
$$

Note that it follows from properties (i) and (ii) that $\Gamma$ is closed under L-provability.

Lemma 2.2 Suppose that $K$ is a Kripke frame of topwidth $n+1$ in which $\varphi_{k}$ does not hold. Then $\left(\begin{array}{c}n \\ \lfloor n / 2\rfloor\end{array}\right) \geq k$.

Proof Under the assumptions, we prove that the power set $\mathcal{P}(\{1, \ldots, n\})$ has an antichain of size $k$. The lemma then follows from Sperner's theorem (see Sperner [16, p. 544]; see also Aigner and Ziegler [1]) stating that $\left(\begin{array}{c}n \\ \lfloor n / 2\rfloor\end{array}\right)$ is the greatest number $k$ for which there is an antichain of $k$ pairwise incomparable subsets of $\{1, \ldots, n\}$.

Since there is a model on the frame $K$ that falsifies $\varphi_{k}$, there must be a maximal node in which $\neg p_{1} \wedge \cdots \wedge \neg p_{k}$ holds. This leaves $n$ nodes to falsify all implications $\neg p_{i} \rightarrow \neg p_{j}$ with $i \neq j$. Label these nodes by $1, \ldots, n$. Let $S_{i} \subseteq\{1, \ldots, n\}$ be the set of nodes where $p_{i}$ holds, with $i=1, \ldots, k$. Then the sets $S_{i}$ form an antichain since for every pair $i \neq j$ there is a node that falsifies $\neg p_{i} \rightarrow \neg p_{j}$ (hence in which $p_{i}$ and $\neg p_{j}$ hold). 
Lemma 2.3 Suppose that $\left(\Gamma_{1}, \Delta_{1}\right), \ldots,\left(\Gamma_{n}, \Delta_{n}\right)$ are distinct maximal nodes in the canonical model of $\mathrm{L}$. Then for every $S \subseteq\{1, \ldots, n\}$ there is a formula $A$ such that $A \in \Gamma_{j}$ if and only if $j \in S$.

Proof By maximality, the $\Gamma_{i}$ are pairwise $\subseteq$-incomparable; hence for every $i \neq j$, there is a formula $A_{i, j} \in \Gamma_{i}-\Gamma_{j}$. Hence, taking $A_{i}=\bigwedge_{j \neq i} A_{i, j}$ for every $i$, it is easy to see that $\left(\Gamma_{i}, \Delta_{i}\right) \Vdash A_{i} \rightarrow \neg A_{j}$ for every $i \neq j$. Now let $A=\bigvee_{j \in S} A_{j}$.

Theorem 2.4 For every $k \geq 1$, IPC $+\varphi_{k}$ is complete with respect to the class of Kripke frames of topwidth at most $n$, where $n$ is minimal such that

$$
\left(\begin{array}{c}
n \\
\lfloor n / 2\rfloor
\end{array}\right) \geq k .
$$

Proof For the right-to-left implication, suppose that $K$ is a frame of topwidth $m+1 \leq n$ in which $\varphi_{k}$ does not hold. Then by Lemma 2.2, $\left(\begin{array}{c}m \\ \lfloor m / 2\rfloor\end{array}\right) \geq k$; hence by minimality of $n$ we have $m \geq n$, which is a contradiction. Hence any frame of topwidth $l \leq n$ satisfies $\varphi_{k}$.

For the converse direction, we have to show that if $\varphi$ is a formula that IPC $+\varphi_{k}$ does not prove, then there is a Kripke frame of topwidth at most $n$, where $n$ and $k$ are related as in the statement of the theorem, in which $\varphi$ does not hold, that is, there is a model on this frame on which $\varphi$ does not hold. We show that a part of the canonical model of IPC $+\varphi_{k}$ has this property.

Now if $\varphi$ is not provable in IPC $+\varphi_{k}$, then the tableau $\left(\left\{\varphi_{k}\right\},\{\varphi\}\right)$ is consistent, but not maximal. By Lindenbaum's lemma (see [4, Lemma 5.1]) it can be extended to a maximal tableau $t=(\Gamma, \Delta)$, which is thus a node in the canonical model in which $\varphi$ does not hold by the property (4). Let $K^{t}$ denote the part of $K$ that is $R$-reachable from $t$. We prove that $K^{t}$ has the required property.

First we note that every node in $K$ is below an $R$-maximal one: every path in $K$ has an upper bound (by taking unions on the first coordinate and intersections on the second); hence an application of Zorn's lemma gives a maximal element above any node in $K$.

We now show that $K^{t}$ has at most $n R$-maximal nodes. Suppose for a contradiction that there exist at least $n+1$ distinct maximal nodes

$$
\left(\Gamma_{1}, \Delta_{1}\right), \ldots,\left(\Gamma_{n+1}, \Delta_{n+1}\right) .
$$

Since $\left(\begin{array}{c}n \\ \lfloor n / 2\rfloor\end{array}\right) \geq k$, there is an antichain $S_{1}, \ldots, S_{k}$ in $\mathcal{P}(\{1, \ldots, n\})$ of size $k$. For every $S_{i}$, with the help of Lemma 2.3 choose a formula $A_{i}$ such that

$$
A_{i} \in \Gamma_{j} \Longleftrightarrow j \in S_{i}
$$

and such that $A_{i} \notin \Gamma_{n+1}$. Note that by maximality it follows from (5) that

$$
\neg A_{i} \in \Gamma_{j} \Longleftrightarrow A_{i} \notin \Gamma_{j} \Longleftrightarrow j \notin S_{i} .
$$

But now we can prove that $\varphi_{k}$ is not forced in $t$. First $t \nVdash \neg\left(\neg A_{1} \wedge \cdots \wedge \neg A_{k}\right)$ because $\left(\Gamma_{n+1}, \Delta_{n+1}\right) \Vdash \neg A_{1} \wedge \cdots \wedge \neg A_{k}$ by choice of $A_{i}$. Also $t \nVdash \neg A_{i} \rightarrow \neg A_{i^{\prime}}$ for every $i \neq i^{\prime}$ with $i, i^{\prime} \leq k$. Namely, the elements $S_{i}$ and $S_{i^{\prime}}$ of the antichain are incomparable; hence $j \in S_{i^{\prime}}-S_{i}$ for some $j \in\{1, \ldots, n\}$. Thus, by definition of $A_{i}$, we have $A_{i^{\prime}} \in \Gamma_{j}$ and $\neg A_{i} \in \Gamma_{j}$, and hence $\left(\Gamma_{j}, \Delta_{j}\right) \Vdash \neg A_{i} \wedge A_{i^{\prime}}$. So we see that $t$ does not force the formula $\varphi_{k}\left(A_{1}, \ldots, A_{k}\right)$ obtained from $\varphi_{k}$ by substituting 
$A_{i}$ for every variable $p_{i}$. But then it follows that $t \nVdash \varphi_{k}$, for if $t \Vdash \varphi_{k}$, then $t$ would also force $\varphi_{k}\left(A_{1}, \ldots, A_{k}\right)$ because we work over the logic IPC $+\varphi_{k}$, which by definition proves every substitution instance of $\varphi_{k}$.

By the property (4), every formula of a logic $L$ holds in the canonical model of $L$. A logic $L$ is called canonical if every formula of $L$ holds in the canonical frame of $L$. Note that the proof of Theorem 2.4 shows that the logics of $\varphi_{k}$ are canonical in this sense.

Following [7, p. 69], a condition $F$ on a partially ordered set $\langle K, R, 0\rangle$ with least element 0 is absolute if it can be formulated in a higher-order language (with symbols for $R, 0,=$ ), and for every $\langle K, R, 0\rangle$ satisfying $F$, there exists a finite $K_{0} \subseteq K$ such that for every $K^{\prime}$, with $K_{0} \subseteq K^{\prime} \subseteq K$, we have that also $\left\langle K^{\prime}, R \backslash K^{\prime}, 0\right\rangle$ satisfies $F$. It is known (see, e.g., [7, p. 69]) that if $L$ is an intermediate logic which characterizes a class of Kripke frames, consisting of exactly the frames satisfying an absolute condition $F$, then $\mathrm{L}$ also characterizes the class of finite Kripke frames satisfying $F$. An intermediate logic $\mathrm{L}$ is said to have the finite model property, if for every $\varphi$ with $\varphi \notin \mathrm{L}$, there exists a finite Kripke model which does not satisfy $\varphi$. By a classical theorem of Harrop (see [8, Lemma 4.1]; see also [7, p. 266]), if an intermediate logic $\mathrm{L}$ has the finite model property and is finitely axiomatizable, then $\mathrm{L}$ is decidable. Therefore, we have the following.

Theorem 2.5 For every $k \geq 1$, IPC $+\varphi_{k}$ is complete with respect to the class of finite Kripke frames with topwidth at most $n$, where $n$ is least such that $\left(\begin{array}{c}n \\ \lfloor n / 2\rfloor\end{array}\right) \geq k$. Moreover, IPC $+\varphi_{k}$ is decidable.

Proof The claim follows by the above-quoted remark and the fact that the condition of being a Kripke frame with topwidth at most $n$, and $n$ least such that $\left(\begin{array}{c}n \\ \lfloor n / 2\rfloor\end{array}\right) \geq k$, is absolute.

Finally, we have the following additional characterization of IPC $+\varphi_{k}$.

Corollary 2.6 We have that IPC $+\varphi_{k}=\mathrm{IPC}+\sigma_{n}$, for all $n$ and $k$ such that $n$ is minimal with $\left(\begin{array}{c}n \\ \lfloor n / 2\rfloor\end{array}\right) \geq k$.

Proof This follows from Theorem 2.4 and Smorynski's completeness theorem.

Note that the sequence of logics IPC $+\varphi_{k}$ is decreasing, but not strictly decreasing, with respect to inclusion. Namely, if $k_{1}<k_{2}$ and $n$ is the least such that $\left(\begin{array}{c}n \\ \lfloor n / 2\rfloor\end{array}\right) \geq k_{1}$, but $n$ is also the least such that $\left(\begin{array}{c}n \\ \lfloor n / 2\rfloor\end{array}\right) \geq k_{2}$, then

$$
\mathrm{IPC}+\varphi_{k_{1}}=\mathrm{IPC}+\varphi_{k_{2}}=\mathrm{IPC}+\sigma_{n} .
$$

\section{Algebraic Semantics}

A Brouwer algebra is an algebra $\langle L,+, \times, \rightarrow, \neg, 0,1\rangle$ where $\langle L,+, \times, 0,1\rangle$ is a bounded distributive lattice (with + and $\times$ denoting the operations of sup and inf, respectively) and $\rightarrow$ is a binary operation satisfying

$$
b \leq a+c \Leftrightarrow a \rightarrow b \leq c,
$$

or, equivalently,

$$
a \rightarrow b=\text { least }\{c: b \leq a+c\},
$$


and $\neg$ is the unary operation, given by $\neg a=a \rightarrow 1$. A Brouwer algebra $L$ satisfies a propositional formula $\sigma$ (denoted by $L \models \sigma$; we also say that $\sigma$ is an identity of $L$ ) if whatever substitution of elements of $L$ in place of the propositional variables of $\sigma$ (interpreting the connectives $\vee, \wedge, \rightarrow, \neg$ with the operations $\times,+, \rightarrow, \neg$, resp.) yields the element 0 . (Note that this definition of truth is dual to that in a Heyting algebra; see also the remarks on Heyting algebras below.) Let

$$
\operatorname{Th}(L)=\{\sigma: L \models \sigma\} .
$$

It is well known that IPC $\subseteq \operatorname{Th}(L)$, for every Brouwer algebra $L$. An intermediate logic $\mathrm{L}$ is complete with respect to a class of Brouwer algebras, if for every formula $\sigma, L$ derives $\sigma$ if and only if every algebra in the class satisfies $\sigma$.

Recall that in a distributive lattice $L$, we have that an element $a \in L$ is joinirreducible if and only if $a \leq x+y$ implies that $a \leq x$ or $a \leq y$, for every $x, y \in L$. Thus if $L$ is a Brouwer algebra, $b \in L$ with $b=\sum X$, where $X$ is a finite set consisting of join-irreducible elements, then for every $a \in L$,

$$
a \rightarrow b=\sum\{x \in X: x \not \leq a\} .
$$

This follows from the fact that $b \leq a+y$, where $y=\sum\{x \in X: x \not \leq a\}$, and by join irreducibility of each element of $X$, we have that $x \leq c$ for every $c$ such that $b \leq a+c$ and every $x \in X$ such that $x \not \leq a$. Thus $y$ is the least such that $b \leq a+y$. Finally, if $X$ is an antichain of join-irreducible elements in a distributive lattice, and $I, J \subseteq X$ are finite sets, then

$$
\sum I \leq \sum J \Leftrightarrow I \subseteq J .
$$

Recall the following well-known construction (see Fitting [6, Chapter 1, Section 6]) which associates with every Kripke frame a Brouwer algebra, whose identities coincide with the formulas that hold in the frame. Let $K$ be a given Kripke frame, with accessibility relation $R$ : a subset $A \subseteq K$ is open, if for every $x, y \in K$ we have that $x \in A$ and $x R y$, then $y \in A$. Let $\operatorname{Op}(A)$ be the collection of open subsets of $K$. The following two lemmas, based on [6], appear in Ono [10].

Lemma 3.1 ([10, Corollary 1.3.1]) The distributive lattice $\operatorname{Alg}(K)=\langle\mathrm{Op}(K),+$, $\times, \rightarrow 0,1\rangle$ is a Brouwer algebra, where $A+B=A \cap B, A \times B=A \cup B$,

$$
A \rightarrow B=\{x \in K:(\forall y \in K)[x R y \wedge y \in A \Rightarrow y \in B]\},
$$

$0=K$, and $1=\emptyset$. Moreover,

$$
\{\varphi: K \models \varphi\}=\{\varphi: \operatorname{Alg}(K) \models \varphi\} .
$$

Proof See [6]. In fact, the theorem in [6] is formulated in terms of Heyting algebras. Recall that $L$ is a Heyting algebra if the dual $L^{\mathrm{op}}$ is a Brouwer algebra. If $L$ is a Heyting algebra, we write $L \models{ }^{H} \sigma$, if $L^{\text {op }} \models \sigma$. In [6] it is shown that the collection of open sets together with the operations $+=\cup, \times=\cap, 0=\emptyset, 1=K$, and

$$
A \rightarrow B=\{x \in K:(\forall y \in K)[x R y \wedge y \in A \Rightarrow y \in B]\},
$$

is a Heyting algebra which satisfies the same formulas as $K$. To prove our result, given a frame $K$, apply Fitting's construction to get a Heyting algebra, and then take its dual: the claim then follows from the obvious fact that the formulas satisfied (under $\models$ ) by a Brouwer algebra are the same as the ones satisfied (under $\models^{H}$ ) by its dual Heyting algebra. 
Conversely, given a Brouwer algebra $L$ with meet-irreducible 0 , let $I(L)$ be the collection of prime ideals of $L$, which becomes a Kripke frame $\operatorname{Kr}(L)=\langle I(L), \subseteq\rangle$. (Note that $\operatorname{Kr}(L)$ satisfies our assumption that all Kripke frames have a root, since $0 \in L$ is meet-irreducible, so that $\{0\}$ is a prime ideal.)

Lemma 3.2 (Ono [10, Corollary 1.5]) For every Brouwer algebra L, we have

$$
\{\varphi: L \models \varphi\} \subseteq\{\varphi: \operatorname{Kr}(L) \models \varphi\} .
$$

Moreover, equality holds if $L$ is finite.

Proof See [10]. Again, a few words may be spent on the proof, since [10] uses Heyting algebras instead of Brouwer algebras. So, suppose that we are given a Brouwer algebra $L$; take its dual $L^{\mathrm{op}}$, which is a Heyting algebra, and then use [10] to conclude that $\left\langle F\left(L^{\mathrm{op}}\right), \subseteq\right\rangle$ (where $F\left(L^{\mathrm{op}}\right)$ is the collection of prime filters of $L^{\mathrm{op}}$ ) is a Kripke frame $K$ that satisfies $\left\{\varphi: L^{\text {op }} \models^{H} \varphi\right\} \subseteq\{\varphi: K \models \varphi\}$, with equality if $L^{\text {op }}$ is finite. The claim then follows from the fact that $\left\{\varphi: L^{\mathrm{op}} \models^{H} \varphi\right\}=\{\varphi: L \models \varphi\}$, and $F\left(L^{\mathrm{op}}\right)$ is order-isomorphic to $I(L)$ under $\subseteq$, as easily follows from recalling that in a distributive lattice $L$, for every $X \subseteq L, X$ is a prime filter if and only if $L-X$ is a prime ideal.

Theorem 2.5 has the following algebraic counterpart.

Theorem 3.3 Each IPC $+\varphi_{k}$ is complete with respect to the class of all finite Brouwer algebras $L$ with meet-irreducible 0 and at most $n$ coatoms, where $n$ is minimal such that $\left(\begin{array}{c}n \\ \lfloor n / 2\rfloor\end{array}\right) \geq k$.

Proof The proof follows from Theorem 2.4 and Lemmas 3.1 and 3.2, together with the following observations.

1. If $K$ has topwidth $n$, then $\operatorname{Alg}(K)$ has $n$ coatoms. Indeed, for every maximal element $x$ in the frame, the singleton $\{x\}$ is open, and this is clearly a coatom in $\operatorname{Alg}(K)$; moreover, the coatoms in $\operatorname{Alg}(K)$ are all of this form.

2. If a finite Brouwer algebra $L$ has $n$ coatoms, then $\operatorname{Kr}(L)$ is of topwidth $n$. Indeed, in a finite Brouwer algebra $L$, the ideals generated by the coatoms are prime and contain all other prime ideals, generated by meet-irreducible elements. In other words, the coatoms correspond exactly to the maximal elements in $\operatorname{Kr}(L)$.

Finally, note that, for every Kripke frame $K, \operatorname{Alg}(K)$ has meet-irreducible 0, since the Kripke frames in this paper always have a least element.

For finite Brouwer algebras, we may also describe the completeness property in terms of join-irreducible elements joining to the greatest element 1 .

Definition 3.4 For every $n$, let $\mathfrak{B}_{n}$ denote the class of Brouwer algebras in which the top element is the join of some antichain of $n$ join-irreducible elements.

Note that in any distributive lattice, if $\sum X=\sum Y$, where $X, Y$ are finite antichains of join-irreducible elements, then it follows from (8) that $X=Y$. Thus, in a finite distributive lattice $L$, or more generally in a distributive lattice $L$ having the finite descending chain condition (see, e.g., Balbes and Dwinger [2, Theorem III.2.2]), each element is the join of a unique antichain of join-irreducibles, and thus $L$ belongs to $\mathfrak{B}_{n}$, for a unique $n$. 
Lemma 3.5 If $L$ is a finite Brouwer algebra, then $L$ has exactly $n$ coatoms if and only if $L \in \mathfrak{B}_{n}$.

Proof Suppose that $L \in \mathfrak{B}_{n}$ is finite, and let $b_{1}, \ldots, b_{n}$ be the antichain of $n$ join-irreducible elements such that $1=\sum_{i=1}^{n} b_{i}$. For every $i$, let $\hat{b}_{i}=\sum_{j \neq i} b_{j}$. We claim that each $\hat{b_{i}}$ is a coatom. Indeed $\hat{b_{i}}<1$, as $b_{i} \not \hat{b}_{i}$; moreover, assume that $\hat{b_{i}} \leq b$, and let $b=\sum X$, where $X$ is an antichain of join-irreducible elements. (Here we use that $L$ is finite.) By join irreducibility, we have

$$
\left\{b_{j}: j \neq i\right\} \subseteq X \subseteq\left\{b_{j}: 1 \leq j \leq n\right\},
$$

and thus either $\hat{b}_{i}=b$ or $b=1$. It follows that $L$ has at least $n$ coatoms. On the other hand, suppose that $L$ has also a coatom $a \notin\left\{\hat{b_{i}}: 1 \leq i \leq n\right\}$. Then for every $i, \hat{b_{i}}+a=1$, thus $b_{i} \leq \hat{b_{i}}+a$; hence by join irreducibility, $b_{i} \leq a$. This implies that $\sum_{i} b_{i} \leq a$; hence $a=1$, which is a contradiction.

Conversely, suppose that $L$ is a finite Brouwer algebra that has $n$ coatoms. Since $L$ is finite, there exists $m$ such that $L \in \mathfrak{B}_{m}$. On the other hand, the above argument shows that $m=n$, so that $L \in \mathfrak{B}_{n}$.

Let $\mathfrak{B}_{n}^{\perp}$ be the subclass of $\mathfrak{B}_{n}$ consisting of the algebras with meet-irreducible 0 . Then we have the following.

Corollary 3.6 Each IPC $+\varphi_{k}$ is complete with respect to the class of finite Brouwer algebras $\mathfrak{B}_{n}^{\perp}$, where $n$ is minimal such that $\left(\begin{array}{c}n \\ \lfloor n / 2\rfloor\end{array}\right) \geq k$.

Proof This is immediate from Theorem 3.3 and Lemma 3.5.

Finally, we prove Theorem 3.8 below, which holds also for Brouwer algebras that are not necessarily finite. We need a preliminary lemma, which illustrates the range of $\neg$ in a Brouwer algebra from $\mathfrak{B}_{n}$.

Lemma 3.7 Let $L \in \mathfrak{B}_{n}$, and let $b_{1}, \ldots, b_{n}$ be an antichain of join-irreducible elements such that $1=b_{1}+\cdots+b_{n}$. Then every negation $\neg$ a in $L$ is of the form $\neg a=\sum_{i \in I} b_{i}$ for some subset $I \subseteq\{1, \ldots, n\}$ (where, of course, $\neg a=0$ if $I=\emptyset$ ). In particular, $\neg b_{i}=\sum_{j \neq i} b_{j}$.

Proof By (7) we have $\neg a=\sum_{i \in I} b_{i}$, where $I=\left\{i: b_{i} \not \leq a\right\}$.

Theorem 3.8 Let $\left(\begin{array}{c}n \\ \lfloor n / 2\rfloor\end{array}\right)=k$. Then the following hold:

(i) if $L \in \mathfrak{B}_{m}$ and $m \leq n$, then $L \models \varphi_{k}$;

(ii) if $L \in \mathfrak{B}_{m}^{\perp}$ and $m>n$, then $L \not \forall \varphi_{k}$.

Proof (i) Let $k$ and $n$ be as in the statement of the theorem. Let $L \in \mathfrak{B}_{m}, m \leq n$, with $b_{1}, \ldots, b_{m}$ join-irreducible elements that join to 1 . In order to show that $\varphi_{k}$ holds in $L$, we take any sequence $a_{i}$ of $k$ elements in $L$ and show that $\varphi_{k}$ evaluates to 0 for $p_{i}=a_{i}$. If there are $i \neq j$ such that $\neg a_{i}$ and $\neg a_{j}$ are comparable, then the first clause of $\varphi_{k}$ is satisfied. So suppose that all $\neg a_{i}$ are pairwise incomparable. We have to show that then the last clause of $\varphi_{k}$ is satisfied, that is, that $\neg\left(\neg a_{1}+\cdots+\neg a_{k}\right)=0$, or equivalently, that $\sum_{i=1}^{k} \neg a_{i}=1$. By Lemma 3.7, every $\neg a$ is of the form $\neg a=\sum_{i \in I} b_{i}$. Note that $\sum_{i \in I} b_{i} \leq \sum_{j \in J} b_{j}$ if and only if $I \subseteq J$, as follows from (8). So to the $k$ incomparable negations $\neg a_{i}$ corresponds a collection of $k$ pairwise $\subseteq$-incomparable subsets of $\{1, \ldots, m\}$. Sperner's theorem 
says that $\left(\begin{array}{c}m \\ \lfloor m / 2\rfloor\end{array}\right)$ is the maximum number $k$ for which there is such an antichain of $k$ pairwise incomparable subsets of $\{1, \ldots, m\}$. Hence because $\left(\begin{array}{c}m \\ \lfloor m / 2\rfloor\end{array}\right) \leq k$, the collection corresponding to the $\neg a_{i}$ covers all of $\{1, \ldots, m\}$, and in particular

$$
\sum_{i=1}^{k} \neg a_{i}=\sum_{i=1}^{m} b_{i}=1,
$$

which is what we had to prove.

(ii) Suppose that $L \in \mathfrak{B}_{m}^{\perp}$, with $m>n$; let

$$
I=\left\{b_{1}, \ldots, b_{n}, b_{n+1}, \ldots, b_{m}\right\}
$$

be an antichain of join-irreducible elements such that in $L$ we have $1=\sum_{1 \leq i \leq m} b_{i}$. By Sperner's theorem, take a collection of $k$ incomparable subsets $\left\{I_{i}: 1 \leq i \leq k\right\}$ of $\{1, \ldots, n\}$. For every $i=1, \ldots, k$ choose $a_{i}$ so that $\neg a_{i}=\sum_{j \in I_{i}} b_{j}$. (The proof of Lemma 3.7 shows how to achieve this: take $a_{i}=\sum_{j \notin I_{i}} b_{j}$.) Then the negations $\neg a_{i}$ are incomparable because the sets $I_{i}$ form an antichain, and hence the first clause of $\varphi_{k}$ is nonzero (as 0 is meet-irreducible in $L$ ). We also have

$$
\sum_{i=1}^{k} \neg a_{i}=\sum_{\substack{1 \leq i \leq k \\ j \in I_{i}}} b_{j} \neq 1
$$

(because no $b_{j}$, with $j>n$, is included); hence $\neg\left(\sum_{i=1}^{k} \neg a_{i}\right) \neq 0$ and the second clause of $\varphi_{k}$ is also nonzero. So $\varphi_{k}$ does not evaluate to 0 in $L$, since in this algebra, 0 is meet-irreducible.

\section{An Application to the Medvedev Lattice}

This section is an addendum to Sorbi and Terwijn [15]. We thank Paul Shafer [11] for pointing out some inaccuracies in that paper. In [15] logics of the form $\mathrm{Th}(\mathfrak{M} / \mathbf{A})$ are studied, where $\mathfrak{M}$ is the Medvedev lattice, $\mathbf{A} \in \mathfrak{M}$, and $\mathfrak{M} / \mathbf{A}$ is the initial segment of $\mathfrak{M}$ consisting of all $\mathbf{B} \in \mathfrak{M}$ such that $\mathbf{B} \leq \mathbf{A}$. The Medvedev lattice arises from the following reducibility on subsets of $\omega^{\omega}$ (also called mass problems): if $\mathcal{A}, \mathscr{B}$ are mass problems, then $\mathcal{A} \leq \mathscr{B}$, if there is an oracle Turing machine which, when given as oracle any function $g \in \mathcal{B}$, computes a function $f \in \mathcal{A}$. The Medvedev degrees, or simply, $M$-degrees, are the equivalence classes of mass problems under the equivalence relation generated by $\leq$. The collection of all M-degrees constitutes a bounded distributive lattice, called the Medvedev lattice, which turns out to be in fact a Brouwer algebra, that is, it is equipped with a suitable operation $\rightarrow$, satisfying (6). Hence every factor of the form $\mathfrak{M} / \mathbf{A}$ is itself a Brouwer algebra, being closed under $\rightarrow$, with $\neg$ given by $\neg \mathbf{B}=\mathbf{B} \rightarrow \mathbf{A}$. In the following we use the notation from [15], to which the reader is also referred for more details and information about the Medvedev lattice and intermediate propositional logics.

In order to show that there are infinitely many logics of the form $\operatorname{Th}(\mathfrak{M} / \mathbf{A})$, in [15] a sequence of M-degrees $\mathbf{B}_{n}, n \in \omega$, is introduced. In [15, Corollary 5.8] it is claimed that the logics $\operatorname{Th}\left(\mathfrak{M} / \mathbf{B}_{n}\right)$ are all different, but no detailed proof of this is given. Below we prove that indeed these logics are all different from each other. In particular, for any $f \in \omega^{\omega}$ consider the mass problem

$$
\mathscr{B}_{f}=\left\{g \in \omega^{\omega}: g \not{ }_{T} f\right\} ;
$$


then the Medvedev degree $\mathbf{B}_{f}$ of $\mathscr{B}_{f}$ is join-irreducible (see Sorbi [13]). Recall that the top element 1 of $\mathfrak{M} / \mathbf{B}_{n}$ is the join

$$
\mathbf{B}_{n}=\mathbf{B}_{f_{1}}+\cdots+\mathbf{B}_{f_{n}},
$$

where $\left\{f_{i}: i \in \omega\right\}$ is a collection of functions whose Turing degrees are pairwise incomparable. In particular, the top element of $\mathfrak{M} / \mathbf{B}_{1}$ is join-irreducible and the top elements of all other factors $\mathfrak{M} / \mathbf{B}_{n}$ are not. Hence $\operatorname{Th}\left(\mathfrak{M} / \mathbf{B}_{1}\right)$ can be distinguished from all the other theories by the formula $\neg p \vee \neg \neg p$. Namely, the w.l.e.m. holds in a factor $\mathfrak{M} / \mathbf{A}$ if and only if $\mathbf{A}$ is join-irreducible (see Sorbi [14]). We recall that the least element of $\mathfrak{M}$, and thus of every factor $\mathfrak{M} / \mathbf{A}$, is meet-irreducible. Hence $\mathfrak{M} / \mathbf{B}_{n} \in \mathfrak{B}_{n}^{\perp}$. (This is in fact enough for the proof below.)

Corollary 4.1 If $m \neq n$, then $\operatorname{Th}\left(\mathfrak{M} / \mathbf{B}_{m}\right) \neq \operatorname{Th}\left(\mathfrak{M} / \mathbf{B}_{n}\right)$.

Proof Assume that $n<m$, and let $k=\left(\begin{array}{c}n \\ \lfloor n / 2\rfloor\end{array}\right)$. Since $\mathfrak{M} / \mathbf{B}_{n} \in \mathfrak{B}{ }_{n}^{\perp}$, by Theorem 3.8, we have that $\varphi_{k} \in \operatorname{Th}\left(\mathfrak{M} / \mathbf{B}_{n}\right)$, but $\varphi_{k} \notin \operatorname{Th}\left(\mathfrak{M} / \mathbf{B}_{m}\right)$. Note also that by Corollary 2.6, we can now also conclude that $\sigma_{n} \in \operatorname{Th}\left(\mathfrak{M} / \mathbf{B}_{n}\right)$, but $\sigma_{n} \notin \operatorname{Th}\left(\mathfrak{M} / \mathbf{B}_{m}\right)$.

\section{References}

[1] Aigner, M., and G. M. Ziegler, Proofs from The Book, 3rd edition, Springer, Berlin, 2004. MR 2014872. DOI 10.1007/978-3-662-05412-3. 323

[2] Balbes, R., and P. Dwinger, Distributive Lattices, University of Missouri Press, Columbia, Mo., 1974. MR 0373985. 327

[3] Brouwer, L. E. J., "Consciousness, philosophy, and mathematics," pp. 1235-49 in Library of the Tenth International Congress of Philosophy (Amsterdam, 1948), edited by E. Beth, H. Pos, and H. Hollak, publisher unknown, 1948. MR 0028260. 321

[4] Chagrov, A., and M. Zakharyaschev, Modal Logic, vol. 35 of Oxford Logic Guides, Oxford University Press, New York, 1997. MR 1464942. 322, 323, 324

[5] Dummett, M. A. E., and E. J. Lemmon, "Modal logics between S4 and S5," Zeitschrift für mathematische Logik und Grundlagen der Mathematik, vol. 5 (1959), pp. 250-64. MR 0156783. 322

[6] Fitting, M. C., Intuitionistic Logic, Model Theory and Forcing, vol. 21 of Studies in Logic and the Foundations of Mathematics, North-Holland, Amsterdam, 1969. MR 0262056. 326

[7] Gabbay, D. M., Semantical Investigations in Heyting's Intuitionistic Logic, vol. 148 of Synthese Library, D. Reidel, Dordrecht, Boston, 1981. MR 0613144. Zbl 0453.03001. 322, 323, 325

[8] Harrop, R., "On the existence of finite models and decision procedures for propositional calculi," Proceedings of the Cambridge Philosophical Society, vol. 54 (1958), pp. 1-13. MR 0093482. 325

[9] Jankov, V. A., "Calculus of the weak law of the excluded middle" (in Russian), Rossiiskaya Akademiya Nauk. Izvestiya Seriya Matematicheskaya, vol. 32 (1968), pp. 1044-51. MR 0237298. 323

[10] Ono, H., "Kripke models and intermediate logics," Publications of the Research Institute for Mathematical Sciences, vol. 6 (1970/71), pp. 461-76. Zbl 0226.02025. MR 0294088. 326, 327

[11] Shafer, P., personal communication, May 2009. 329

[12] Smorynski, C. A., Investigations of intuitionistic formal systems by means of Kripke models, Ph.D. dissertation, University of Illinois at Chicago, Chicago, 1973. MR 2623927. 322, 323 
[13] Sorbi, A., "Embedding Brouwer algebras in the Medvedev lattice," Notre Dame Journal of Formal Logic, vol. 32 (1991), pp. 266-75. MR 1123000. DOI 10.1305/ndjfl/1093635751. 330

[14] Sorbi, A., "Some quotient lattices of the Medvedev lattice," Mathematical Logic Quarterly, vol. 37 (1991), pp. 167-82. Zbl 0702.03021. MR 1155135. DOI 10.1002/malq.19910370905. 330

[15] Sorbi, A., and S. A. Terwijn, "Intermediate logics and factors of the Medvedev lattice," Annals of Pure and Applied Logic, vol. 155 (2008), pp. 69-86. Zbl 1156.03026. MR 2455558. DOI 10.1016/j.apal.2008.03.002. 329, 331

[16] Sperner, E., "Ein Satz über Untermengen einer endlichen Menge," Mathematische Zeitschrift, vol. 27 (1928), pp. 544-8. MR 1544925. DOI 10.1007/BF01171114. 323

\section{Acknowledgments}

Thanks go to Paul Shafer for his comments on the paper [15]. We thank Lev Beklemishev for remarks about KC, Wim Veldman for the reference to Brouwer, and Rosalie Iemhoff for general discussions about IPC.

Sorbi

Dipartimento di Ingegneria dell'Informazione e scienze matematiche

University of Siena

53100 Siena

Italy

sorbi@unisi.it

Terwijn

Department of Mathematics

Radboud University Nijmegen

6500 GL Nijmegen

The Netherlands

terwijn@math.ru.nl 